\title{
NUMERICAL RANGES OF DERIVATIONS
}

\author{
by J. KYLE
}

(Received 1st November 1976)

\section{Introduction}

In this paper we shall examine the relationship between the numerical range of an inner derivation, and that of its implementing element.

Much of this paper is taken from the author's doctoral thesis (5) written at the University of Newcastle upon Tyne with the helpful guidance of Professor B. E. Johnson. The author also acknowledges the financial support of the Science Research Council during the period of this research.

In what follows $\mathscr{A}$ will always denote a complex unital Banach algebra, with identity $I$. Given $A$ in $\mathscr{A}$, the (inner) derivation implemented by $A$ is the linear mapping which sends $X$ to $X A-A X$. This will be denoted by $\Delta_{A}$.

We begin by giving the relevant definitions needed for this paper. A more detailed account of numerical ranges in normed algebras may be found in the two monographs by Bonsall and Duncan (2) and (3).

Given a Banach algebra $\mathscr{A}$, the unit sphere of $\mathscr{A}$ will be denoted by $S(\mathscr{A})$. The numerical range of $A$ in $\mathscr{A}$ is then given by

$$
V(A ; \mathscr{A})=\cup\{f(A X): f \in D(X ; \mathscr{A})\}
$$

where the union is taken over all $X$ in $S(\mathscr{A})$, and

$$
D(X ; \mathscr{A})=\left\{f \in \mathscr{A}^{*}: f(X)=1=\|f\|\right\} .
$$

It is known that the numerical range is also given by

$$
V(A ; \mathscr{A})=\{f(A): f \in E(\mathscr{A})\}
$$

where $E(\mathscr{A})$ denotes the set of normalised states of $\mathscr{A}$

$$
\text { (i.e. } \left.\quad E(\mathscr{A})=\left(f \in \mathscr{A}^{*}: f(I)=1=\|f\|\right\}\right) \text {. }
$$

Using this last characterisation it follows easily that $V(A ; \mathscr{A})$ is always a non-empty, compact, convex set in $C$.

When $\mathscr{A}=\mathscr{L}(\mathscr{X})$, for some Banach space $\mathscr{X}$, we may also define the spatial numerical range of $A$, which is given by

$$
W(A ; \mathscr{L}(\mathscr{X}))=\left\{f(A x): f \in \mathscr{X}^{*}, \quad x \in \mathscr{Z} \quad \text { and } \quad\|f\|=\|x\|=1=f(x)\right\} .
$$

Proofs of the following may be found in (2):

$$
\begin{aligned}
& \overline{\operatorname{co}} W(A ; \mathscr{L}(\mathscr{X}))=V(A ; \mathscr{L}(\mathscr{X})) \\
& \overline{\operatorname{co}} \operatorname{Sp}(A ; \mathscr{A}) \subseteq V(A ; \mathscr{A})
\end{aligned}
$$

(where $\operatorname{Sp}(A ; \mathscr{A})$ denotes the spectrum of $A$ in $\mathscr{A}$.) 
An element $\boldsymbol{A}$ in $\mathscr{A}$ is said to be Hermitian whenever

$$
V(A ; \mathscr{A}) \subseteq R,
$$

and is said to be normal whenever

$$
A=H+i K
$$

where $H$ and $K$ are commuting Hermitian elements of $\mathscr{A}$.

Finally we note the following description of $V(A ; \mathscr{A})$.

Proposition 1.1 $V(A ; \mathscr{A})=\cap_{z \in C}\{\lambda \in C:|\lambda-z| \leqslant\|A-z I\|\}$.

Proof. This may be found in (1, Chapter I, §10).

2. Numerical ranges when $\mathscr{A}=\mathscr{L}(\mathscr{X})$.

It is already known that

$$
\operatorname{Sp}\left(\Delta_{A} ; \mathscr{L}(\mathscr{A})\right)=\operatorname{Sp}(A ; \mathscr{A})-\operatorname{Sp}(A ; \mathscr{A})
$$

whenever $\mathscr{A}=\mathscr{L}(\mathscr{X})$, for some Banach space $\mathscr{X}$. (See (5) or (7), where an earlier proof was given.)

In this section we shall obtain the corresponding result for numerical ranges, and examine some of its consequences. Since the techniques apply equally well we shall state the main result for the slightly more general operator $\Gamma_{A, B}$ given by

$$
\Gamma_{A . B}(X)=A X+X B
$$

for all $X$ in $\mathscr{L}(\mathscr{X})$. Proof of the following lemma may be found in (2).

Lemma 2.1. For any Banach algebra $\mathscr{A}$,

$$
V(A ; \mathscr{A})=V\left(L_{A} ; \mathscr{L}(\mathscr{A})\right)=V\left(R_{A} ; \mathscr{L}(\mathscr{A})\right)
$$

where $L_{A}(X)=A X$ and $R_{A}(X)=X A$.

Lemma 2.2. For $A, B$ in the Banach algebra $\mathscr{A}$ we have

$$
V\left(\Gamma_{A, B} ; \mathscr{L}(\mathscr{A})\right) \subseteq V(A ; \mathscr{A})+V(B ; \mathscr{A}) .
$$

Proof. $\quad V\left(\Gamma_{A, B} ; \mathscr{L}(\mathscr{A})\right)=\left\{f\left(\Gamma_{A, B}\right): f \in E(\mathscr{L}(\mathscr{A}))\right\}$

$$
\begin{aligned}
& =\left\{f\left(L_{A}+R_{B}\right): f \in E(\mathscr{L}(\mathscr{A}))\right\} \\
& \subseteq\left\{f\left(L_{A}\right): f \in E(\mathscr{L}(\mathscr{A}))\right\}+\left\{f\left(R_{B}\right): f \in E(\mathscr{L}(\mathscr{A}))\right\} \\
& =V\left(L_{A} ; \mathscr{L}(\mathscr{A})\right)+V\left(L_{B} ; \mathscr{L}(\mathscr{A})\right) \\
& =V(A ; \mathscr{A})+V(B ; \mathscr{A}) .
\end{aligned}
$$

(by Lemma 2.1 ). When $\mathscr{A}=\mathscr{L}(\mathscr{X})$, we also have the reverse inclusion.

Theorem 2.3. Let $\mathscr{A}=\mathscr{L}(\mathscr{X})$, for some Banach space $\mathscr{X}$, and let $\Gamma_{A, B}$ be as above. 
Then

$$
V\left(\Gamma_{A, B} ; \mathscr{L}(\mathscr{L}(\mathscr{X}))\right)=V(A ; \mathscr{L}(\mathscr{X}))+V(B ; \mathscr{L}(\mathscr{X}))
$$

Proof. Lemma 2.2 provides one inclusion. To prove the reverse inclusion, choose $\lambda$ in $W(A ; \mathscr{L}(\mathscr{X}))$ and $\mu$ in $W(B ; \mathscr{L}(\mathscr{X}))$. Then we can find functionals $f$ and $g$ in $\mathscr{X}^{*}$, and $x, y$ in $\mathscr{Z}$ such that

$$
\|f\|=\|x\|=f(x)=1 \quad \text { with } f(A x)=\lambda
$$

and

$$
\|g\|=\|y\|=g(y)=1 \quad \text { with } g(B y)=\mu .
$$

Define $F$ in $\mathscr{L}(\mathscr{X})^{*}$ by

$$
F(T)=f(T y) \text { for all } T \text { in } \mathscr{L}(\mathscr{X}),
$$

and let $X$ be the rank-one operator in $\mathscr{L}(\mathscr{X})$ given by

$$
X z=g(z) x \text { for all } z \text { in } \mathscr{X} \text {. }
$$

Then clearly $\|F\| \doteq\|X\|=1$ and

$$
F(X)=f(X y)=f(g(y) x)=g(y) f(x)=1 .
$$

Finally, we have

$$
\begin{aligned}
F\left(\Gamma_{A, B}(X)\right) & =F(A X+X B) \\
& =f(A X y)+f(X B y) \\
& =f(g(y) A x)+f(g(B y) x) \\
& =f(A x) g(y)+f(x) g(B y) \\
& =\lambda+\mu
\end{aligned}
$$

i.e. $\quad \lambda+\mu \in W\left(\Gamma_{A, B} ; \mathscr{L}(\mathscr{L}(\mathscr{X}))\right)$.

Noting that the convex hull of the sum of two sets is the sum of the convex hulls of the sets, we have

$$
\begin{aligned}
V\left(\Gamma_{A, B} ; \mathscr{L}(\mathscr{L}(\mathscr{X}))\right) & =\overline{\operatorname{co}} W\left(\Gamma_{A, B} ; \mathscr{L}(\mathscr{L}(\mathscr{X}))\right) \\
& \supseteq \operatorname{co}\{W(A ; \mathscr{L}(\mathscr{X}))+W(B ; \mathscr{L}(\mathscr{X}))\} \\
& =\overline{\operatorname{co}}\{W(A ; \mathscr{L}(\mathscr{X}))\}+\overline{\operatorname{co}}\{W(B ; \mathscr{L}(\mathscr{X}))\} \\
& =V(A ; \mathscr{L}(\mathscr{X}))+V(B ; \mathscr{L}(\mathscr{X})),
\end{aligned}
$$

and the proof is complete.

We now examine several consequences of Theorem 2.3.

Corollary 2.4. (i) $V\left(\Delta_{A} ; \mathscr{L}(\mathscr{L}(\mathscr{X}))\right)=V(A ; \mathscr{L}(\mathscr{X}))-V(A ; \mathscr{L}(\mathscr{X}))$.

(ii) Whenever $H$ is Hermitian in $\mathscr{L}(\mathscr{Q})$

$$
\nu\left(\Delta_{H} ; \mathscr{L}(\mathscr{L}(\mathscr{X}))\right)=2 \inf \{(\nu(H ; \mathscr{L}(\mathscr{X}))-\lambda): \lambda \in C\},
$$

where $\nu(A ; \mathscr{A})$ denotes the numerical radius of $A$ in $\mathscr{A}$. 
Corollary 2.5. Suppose $A \in \mathscr{L}(\mathscr{X})$ and $A X=X A$ for all $X$ in $\mathscr{L}(\mathscr{X})$, then $A=\lambda I$ for some $\lambda \in C$.

Proof. We then have

$$
0=V\left(\Delta_{A} ; \mathscr{L}(\mathscr{L}(\mathscr{X}))\right)=V(A ; \mathscr{L}(\mathscr{X}))-V(A ; \mathscr{L}(\mathscr{X}))
$$

which can only happen when $V(A ; \mathscr{L}(\mathscr{L}))$ is a singleton, say $\{\lambda\}$. Then

$$
V(A-\lambda I ; \mathscr{L}(\mathscr{Z}))=V(A ; \mathscr{L}(\mathscr{X}))-\lambda=\{0\}
$$

and so $A=\lambda I$. (This follows from (2) Chapter 1, §4.1.)

Corollary 2.5 is, of course, well known. However, as far as we know this method of proof has never been used before, and we include it here for completeness, since it is quoted below in the proof of Theorem 2.7.

Corollary 2.6. For $A$ in $\mathscr{L}(\mathscr{Q}), \Delta_{A}$ is Hermitian if, and only if, $(A-\lambda I)$ is Hermitian for some $\lambda$ in $C$.

The corresponding result when $A$ is normal is slightly more difficult.

Theorem 2.7. For $A$ in $\mathscr{L}(\mathscr{X}), \Delta_{A}$ is normal if, and only if, $A$ is normal.

Proof. Suppose that $A=H+i K$ with $H$ and $K$ both Hermitian and $H K=K H$. Then

$$
\Delta_{A}=\Delta_{H}+i \Delta_{K}
$$

By Corollary 2.6, both $\Delta_{H}$ and $\Delta_{K}$ are Hermitian, and

$$
\begin{aligned}
\Delta_{H} \Delta_{K}(X) & =\Delta_{H}(X K-K X) \\
& =X K H-K X H-H X K+H K X \\
& =X H K-K X H-H X K+K H X \\
& =\Delta_{K}(X H-H X)=\Delta_{K} \Delta_{H}(X)
\end{aligned}
$$

and so $\Delta_{A}$ is normal in $\mathscr{L}(\mathscr{L}(\mathscr{X}))$.

Suppose now that $\Delta_{A}$ is normal in $\mathscr{L}(\mathscr{L}(\mathscr{X}))$. Then

$$
\Delta_{A}=\Phi+i \Psi
$$

where $\Phi$ and $\Psi$ are Hermitian and $\Phi \Psi=\Psi \Phi$. Now define an operator $H$ on $\mathscr{X}$ by

$$
H(X x)=-\Phi(X P) x
$$

where $X$ is in $\mathscr{L}(\mathscr{X}), x$ in $\mathscr{X}$ is a fixed non-zero vector, and $P$ is a projection, of norm one, onto the one-dimensional subspace spanned by $x$. Then $H$ is well defined on $\mathscr{X}$ (if $X x=0$, then $X P=0$, and so $\Phi(X P)=0$ ). Also $H$ is clearly linear and bounded, i.e. $H \in \mathscr{L}(\mathscr{X})$. Similarly, we may define $K$ in $\mathscr{L}(\mathscr{X})$ by

$$
K(X x)=-\Psi(X P) x \text { for all } X \text { in } \mathscr{L}(\mathscr{Q}) .
$$

Then we have 


$$
\begin{aligned}
{\left[\Delta_{H}(X) Y-\Phi(X) Y\right] x } & =X H Y x-H X Y x-\Phi(X) Y x \\
& =-X \Phi(Y P) x+\Phi(X Y P) x-\Phi(X) Y P x \\
& =\left[-\delta^{2} \Phi(X, Y P)\right] x
\end{aligned}
$$

(Here $\delta^{2}$ is the two-dimensional cobounding operator. See (4) for further details.) Similarly,

$$
\left[\Delta_{K}(X) Y-\Psi(X) Y\right] x=\left[-\delta^{2} \Psi(X, Y P)\right] x
$$

Thus

$$
\begin{aligned}
{\left[\Delta_{(H+i K)}(X) Y-(\Phi+i \Psi)(X) Y\right] x } & =-\left[\delta^{2}(\Phi+i \Psi)(X, Y P)\right] x \\
& =-\left[\delta^{2}\left(\Delta_{A}\right)(X, Y P)\right] \\
& =0
\end{aligned}
$$

(since the cobounding operator annihilates every derivation.)

i.e.

$$
\left[\Delta_{(H+i K)}(X)-\Delta_{A}(X)\right] Y X=0 \text { for all } X, Y \text { in } \mathscr{L}(\mathscr{X})
$$

and so $\Delta_{A}=\Delta_{H}+i \Delta_{K}$.

We now show that $H$ and $K$ are both Hermitian. We have

$$
\begin{aligned}
W(H ; \mathscr{L}(\mathscr{X})) & =\{f(H y):\|f\|=\|y\|=f(y)=1\} \\
& =\{f(H X x):\|f\|=\|X x\|=f(X x)=1\} \\
& =-\{f(\Phi(X P) x):\|f\|=\|X x\|=f(X x)=1\}
\end{aligned}
$$

Now the functional $F$ in $\mathscr{L}(\mathscr{X})^{*}$ given by $F(T)=f(T x)$, takes the value 1 at $X P$ whenever $f(X x)=1$ and so $F(\Phi(X P))$ lies in $W(\Phi ; \mathscr{L}(\mathscr{L}(\mathscr{L})))$ whenever $f(X x)=1$ i.e.

$$
W(H ; \mathscr{L}(\mathscr{X})) \subseteq-W(\Phi ; \mathscr{L}(\mathscr{L}(\mathscr{X}))) \subseteq R
$$

since $\Phi$ is Hermitian.

A similar argument shows that $K$ is also Hermitian. Finally, by the uniqueness of real and imaginary parts, we have

$$
\Delta_{H}=\Phi \quad \text { and } \Delta_{K}=\Psi
$$

and so

i.e.

$$
\Delta_{H} \Delta_{K}=\Delta_{K} \Delta_{H}
$$$$
\Delta_{(H K-K H)}=0 \text {. }
$$

Corollary 2.5 then shows that $H K-K H=\lambda I$, for some $\lambda$ in $C$. But the only scalar multiple of $I$ which can be a commutator is 0 . Hence $H$ and $K$ commute, and $(H+i K)$ is normal. Then

$$
\Delta_{A-(H+i K)}=0
$$

and so $A=H+i K+\mu I$ for some $\mu$ in $C$ and $A$ is normal.

As a comparison for Theorem 2.7, we have:

Proposition 2.8. Let $\mathscr{A}$ be a $W^{*}$-algebra with centre $\mathscr{Z}$. Then $\Delta_{A}$ is normal in $\mathscr{L}(\mathscr{A})$ if, and only if, $A$ is normal in $\mathscr{A}$. 
Proof. As in Theorem 2.7, we can easily show that $\Delta_{A}$ is normal in $\mathscr{L}(\mathscr{A})$ whenever $A$ is normal in $\mathscr{A}$.

On the other hand suppose we can find $\Phi$ and $\Psi$ in $\mathscr{L}(\mathscr{A})$, both Hermitian, such that

$$
\Phi \Psi=\Psi \Phi \text { and } \Delta_{A}=\Phi+i \Psi
$$

By a result due to Sinclair (8), we can find $H_{j}$ and $A_{j}(j=1,2)$ in $\mathscr{A}$ such that

$$
\begin{aligned}
& \Phi=L_{H_{1}}+\Delta_{A_{1}} \\
& \Psi=L_{H_{2}}+\Delta_{A_{2}}
\end{aligned}
$$

with $H_{j}, A_{j}(j=1,2)$ all Hermitian. Then

$$
\Delta_{A}=L_{\left(H_{1}+i H_{2}\right)}+\Delta_{\left(A_{1}+i A_{2}\right)}
$$

Thus $L_{\left(H_{1}+i H_{2}\right)}$ is a derivation, which can only be 0 . Hence $H_{1}+i H_{2}=0$, and so $H_{1}=H_{2}=0$.

i.e.

$$
\Delta_{A}=\Delta_{A_{1}}+i \Delta_{A_{2}}
$$

and so $\Phi=\Delta_{A_{1}}$ and $\Psi=\Delta_{A_{2}}$.

Also, as in Theorem 2.7, we have

$$
\Delta_{\left(A_{1} A_{2}-A_{2} A_{1}\right)}=0 \quad \text { and so }\left(A_{1} A_{2}-A_{2} A_{1}\right)=Z \in \mathscr{Z}
$$

Thus

$$
\Delta_{A_{1}}^{2}\left(A_{2}\right)=0
$$

and so

$$
\Delta_{A_{1}}^{n}\left(A_{2}^{n}\right)=(n !)\left(\Delta_{A_{1}}\left(A_{2}\right)\right)^{n} \quad \text { for all } n
$$

(see (1) Chapter II.18.4). Hence

$$
\begin{aligned}
\left\|Z^{n}\right\|^{1 / n} & =\left\|\left(\Delta_{A_{1}}\left(A_{2}\right)\right)^{n}\right\|^{1 / n} \\
& \leqslant(n !)^{-1 / n}\left\|\Delta_{A_{1}}\right\| \cdot\left\|A_{2}\right\| \\
& \rightarrow 0 \quad \text { as } n \rightarrow \infty
\end{aligned}
$$

i.e. $Z$ is quasi-nilpotent in $\mathscr{Z}$ and so $Z=0$. Thus $A_{1} A_{2}=A_{2} A_{1}$ and $A-\left(A_{1}+i A_{2}\right) \in \mathscr{Z}$, i.e. $A$ is normal as required.

\section{Concluding Remarks}

It has already been shown (6) that when $\mathscr{A}$ is a $W^{*}$-algebra, the spectrum of $\Delta_{A}$ depends directly on the centre of $\mathscr{A}$. A similar connection can be established for numerical ranges, and we hope to explore this area in a forthcoming paper. 


\section{REFERENCES}

(1) F. F. Bonsall and J. DunCAN, Complete normed algebras (Springer-Verlag, 1973).

(2) F. F. BONSALL and J. DUNCAN, Numerical ranges of operators and of elements of normed algebras (Lond. Math. Soc. Lecture Note Series (2), Cambridge University Press, 1971).

(3) F. F. Bonsall and J. Duncan,|Numerical ranges II (Lond. Math. Soc. Lecture Note Series (10), Cambridge University Press, 1973).

(4) B. E. Johnson, Cohomology in Banach algebras, Mem. Amer. Math. Soc. 127 (1972).

(5) J. KYLE, Norms, spectra and numerical ranges of derivations, Ph.D. thesis (University of Newcastle upon Tyne, 1976).

(6) J. KyLE, Spectra of derivations, Math. Proc. Cambridge Philos. Soc. 82 (1977), 49-57.

(7) G. Lumer and M. Rosemblum, Linear operator equations, Proc. Amer. Math. Soc. 10 (1959), 32-41.

(8) A. M. SINCLAIR, Jordan homomorphisms and derivations on semi-simple Banach algebras, Proc. Amer. Math. Soc. 24 (1970), 209-214.

UNIVERSITY OF TECHNOLOGY

LOUGHBOROUGH

LEICESTERSHIRE

ENGLAND.

Present address:

UNIVERSITY OF BIRMINGHAM

BIRMINGHAM

ENGLAND. 